\title{
Ontology-based interactive animation/game generation for Chinese shadow play preservation
}

\author{
Hui Liang ${ }^{1}$, Shujie Deng ${ }^{1}$, Jian Chang $^{1}{ }^{*}$, Jian Jun Zhang ${ }^{1}$, Can Chen ${ }^{2}$, Ruofeng Tong ${ }^{3}$ \\ ${ }^{1}$ Centre for Computer Animation, Bournemouth University, Poole, BH12 5BB, UK \\ ${ }^{2}$ Changzhou University, Changzhou, 213164, China \\ ${ }^{3}$ Zhejiang University, Hangzhou, 310058, China
}

\begin{abstract}
Interactive animation generation is an attractive area of research for current animation developers in the field of virtual reality. As an essential tool for solving problems in many research areas, using standardised and structured terminologies, an ontological analysis could concisely describe the core logic of complex systems at a high level and facilitate system design.

We propose a semantic framework using ontological methods to model the construction of interactive animation and promote integration process in a systematic and standardised way. A usage example is presented to provide guidance in generating animation of hand gesture based interactive Chinese shadow puppetry play for the heritage preservation of the traditional art. Domain-specific ontologies for the Chinese traditional shadow play and hand-gesture-based interaction are developed as the ontological implementation of the framework. Finally, an animation of hand-gesture-based interactive shadow play is generated.
\end{abstract}

Keywords-ontology; hand gesutre based; interactive animation; shadow play; virtual reality; culture heritage preservation

\section{INTRODUCTION}

As one of the most famous Chinese folk arts, traditional shadow play has a long history and contains rich cultural elements. Mainly made of donkey's hide, flat shadow puppet consists of several parts and its joints are connected by threads. When playing, puppeteers use their hands to manipulate the flat shadow puppets through sticks attached on the puppets, and the shadows are projected on a simple rear-illuminated cloth screen to create moving pictures [1], as illustrated in Fig. 1.
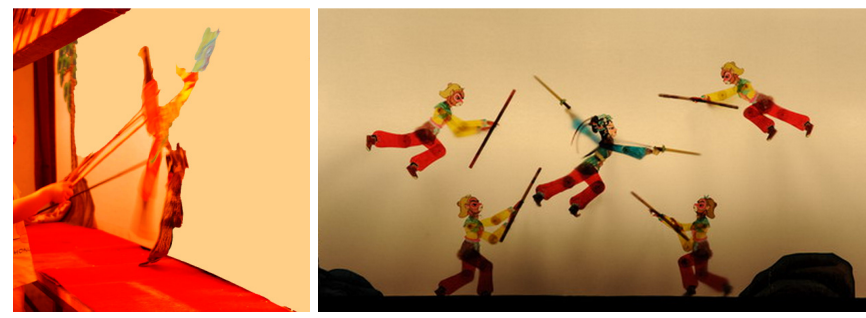

Fig. 1. Chinese traditonal shadow play

However, this intricate operating skill is a higher entry barrier for performers, which requires long time special training. This valuable and fascinating art form is becoming less known to the public, or even dying nowadays. New technologies are required urgently to give new life to the historic art forms.
Our motivation is to develop a novel method to generate interactive shadow puppetry animation for the purpose of cultural heritage preservation and dissemination. The ease of use and user friendly virtual interaction would attract more people's interests and engage young generations to generate shadow play animation without special trainings.

Developed for decades, as an essential technology, interactive animation is widely used in the fields of 3D games and virtual reality by providing users with an immersive experience using natural and intuitive interactions through smart devices in virtual environments, such as touch screens, haptic devices, head mounted devices, motion sensors and tracking devices etc. However, even though advanced theories, techniques, methods, and tools help a lot for generating animation, interactive animation production is still a tedious work and can also be labour-intensive. Its inherent features of complexity - having to handle various aspects of functional requirements (e.g., graphics, physics, artificial intelligence, engineering, multimodal inputs and outputs) - throw out challenges to current research: how can we arrange the plethora of possible combinations of multiple approaches mentioned above?

One appealing solution is leveraging ontology to construct a systematic and standardised framework to describe at a highly abstract and semantic level to provide a full view and understanding of the complex systematic procedure. Using structured terminology, ontological analysis could capture the core logic of complex system with natural language descriptions [2]. In recent years, as a high-level conceptual specification, the notion of ontologies has become increasingly important in the computer animation related domains, such as semantic 3D content representation [3-6], ontology-based 3D model retrieval [7-8] and their usage in virtual scene and game environment (e.g., modelling the semantic information of the virtual game environment).

Additionally, animation data management has become a focal attention to animation production and a popular issue of interests including animation data archiving and reuse in a more efficient and user-friendly way. Because of the phenomenal growth of the animation data, efficient data management, such as structured data presentation and searching for particular information, has become a daunting task. Traditional text-based or content-based media data retrieval introduces problems of the semantic gap between the low level description and the high level semantic interpretation of multimedia object. Ontology-based semantic retrieval, as an

*Corresponding author:jchang@bournemouth.ac.uk 
appropriate way to represent structured knowledge bases, enables data sharing, reuse and inference, and also advances in bridging the semantic gap [7-8]. It provides us suitable techniques on the animation assets management to facilitate data storage, organization, retrieval, reuse and repurposing.

For the purposes of heritage preservation, and also as a usage example, a hand-gesture-based interactive puppetry animation is generated based on the proposed framework and ontologies. It is expected that our method can provide guidance for building and rapid prototyping of diverse interactive animation/game applications.

Our work has the following contributions:

- A semantic framework for interactive animation generation

- Domain-specific ontologies for interactive animation generation, including ontologies for HCI design, and animation data assets

- Hand-gesture-based interactive animation of traditional Chinese shadow play

The rest of this paper is organised as follows. Section 2 reviews the related work briefly. Semantic framework is proposed in section 3. In Section 4, two domain-specific ontologies are implemented based on the framework, and a hand-gesture-based interactive animation of shadow play is generated as the usage example. Section 5 concludes the paper.

\section{RELATED WORK}

According to Gruber [9], ontology is an explicit formal specification of a conceptualization, which is an effective tool to describe general concepts of entities as well as their properties, relationships and constraints [10]. Also as a practical application in information science and technology, ontology provides the vocabulary to define terminology and the constraints required by different applications that cover various fields, from knowledge engineering to software engineering, taking the advantage of the establishment of common vocabulary and semantic term interpretation [11-12].

Traditional model retrieval is based on keywords or the shape properties similarity (e.g., geometry and topology) [1314], in which only the textual or physical information is utilized. The semantic information is ignored and the efficacy of retrieval has not been satisfactory, however. AIM@SHAPE Network of Excellence [15] made first step of using semantics approach to describe and search 3D models to facilitate reuse of the animation contents [16]. There are similar semantic search engines including 3D model Search Engine (Princeton University) [17] and Google 3D Warehouse [18]. To describe media data such as images, audio, video and 3D objects, ontological solutions, such as the Ontology for Media Resources [19] and the Core Ontology for Multimedia (COMM) [20], are devised on the basis of standard models for data interchange on the Web (e.g., RDF, RDFS, OWL and MPEG-7). To facilitate 3D content accessing, ontology-based approaches are used for semantic modelling of the different 3D models (e.g., geometry, appearance and behaviour). Ontology for virtual human was proposed in [21], incorporating semantics into human shapes.

Another application is to model semantic information of virtual environment. Unlike traditional virtual environments design, semantic virtual environments maintain richer semantic information, in which the ontological model provides an abstract and semantic description that is adequate for computer processing [22]. Different aspects of the conceptual representation of the virtual world are modelled by ontologies as high level and semantic description, including the environment structures, entities' behaviour and domain knowledge [23]. The concept of annotated environment together with structured representations of its contents and purposes is firstly proposed by Thalmann et al in [24]. An ontology-based framework with a cognitive middle layer and environment managed semantic concepts are presented by Chang et al.[25]. Studies [26-27] describe the semantics of a $3 \mathrm{D}$ scene focusing on the high-level description of objects or the composition of existing objects. There are also some ontological research works using semantic information to model the interaction of virtual environments, for example, NiMMiT (Notation for MultiModal interaction Techniques), which is a diagram based notation describing multimodal interaction [28]. A contextual augmented reality environment is proposed in [29], which consists of three elements: the trackables, content objects and interfaces. With the model of SCM (Semantic Content Model) designed for semantic representation of the interactive $3 \mathrm{D}$ contents, the virtual environments are represented at different levels of abstraction: Conceptual level, Concrete level and Platform level [3].

There are also some ontological applications in the domain of computer games. For example, a game ontology that is developed based on game theories to facilitate interactions in multi-agent system [30]; game content ontology [31]; using ontology to describe games characteristics, properties and design process [32-33]; using ontological framework as the guide to develop and employ game-based training [34]; using ontology and a set of rules to represent game logics [35]; ontologies as a solution to enable transfer of meaningful game information between different video games [36]; using defined learning game ontology as the evaluation methodology of serious games for teachers/trainers to choose and retrieve learning games [37]. In Semantic Class Library designed for semantic 3D game virtual environment [38], 3D model can be classified to provide additional semantic information, such as physical attributes, functional information besides 3D representations.

\section{SEMANTIC FRAMEWORK FOR INTERACTIVE ANIMATION}

\section{A. Architecture of interactive animation production process}

Enhanced by the improvement of computer system and interactive devices performance, interactive animation technologies are widely used in computer animation production and game industry. As a complex system, interactive animation involves a plethora multimodal function combinations, such as HCI methods, animation assets etc. As a formal semantic foundation, three-layer architecture is proposed to provide a clear vision of the interactive animation production process, as illustrated in Fig. 2. 

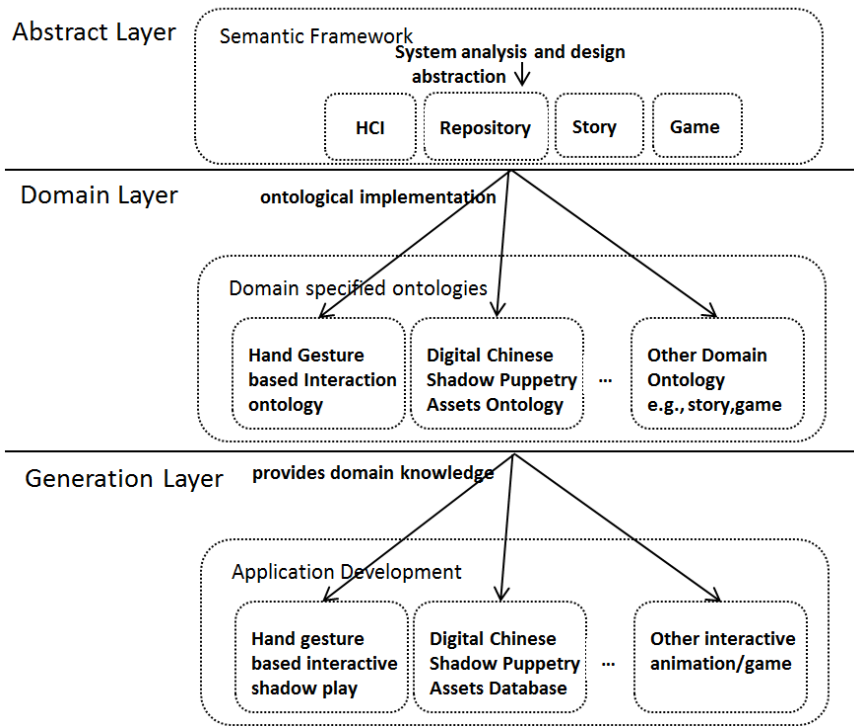

Fig. 2. Architecture of interactive animation production process

\section{B. Semantic framework of interactive animation generation}

Fig. 3 illustrates the components of our semantic framework of interactive animation production. Two things need to be clarified: 1) Our work mainly focuses on two components: HCI and Animation Data Repository rather than including all the technique details, such as story, algorithm programming and system integration; 2) Being constructed at a high abstract semantic level, this framework can be applied to different types of VR interactions development.

In the component of $\mathrm{HCI}$, player interacts with computer system, which involves the use of various input/output devices and interactive modes, such as monitor screen, keyboard, mouse, touch screen, haptic device, motion sensor and tracking device etc. The Animation Data Repository component provides data supports, in which various animation data resources are maintained and managed for the purpose of enhancing reusability.

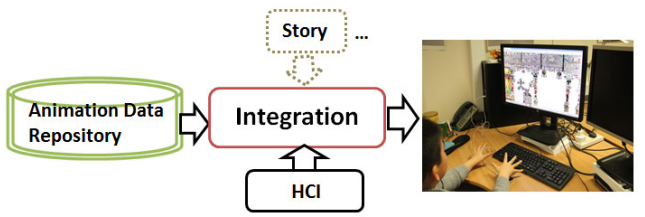

Fig. 3. Semantic framework

\section{1) $\mathrm{HCI}$}

As illustrated in Fig. 4, the HCI component contains a set of structural concepts, which could be decomposed into three levels of semantic abstraction.

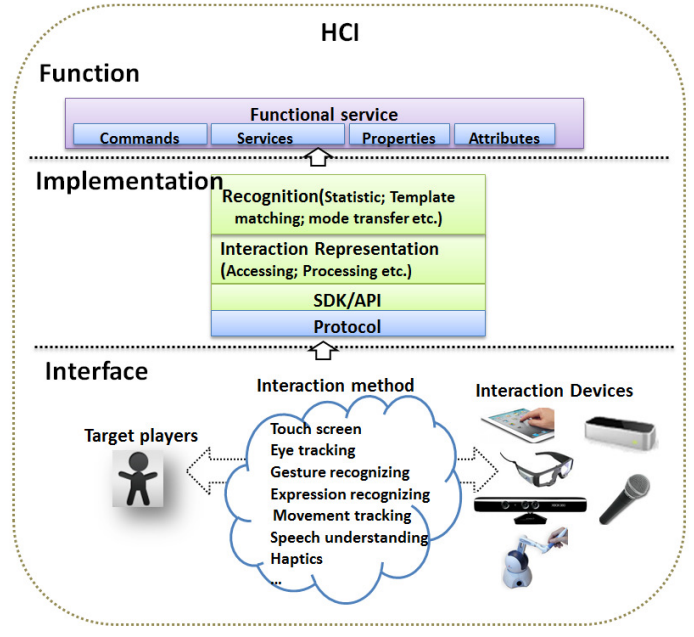

Fig. 4. The HCI component

\section{2) Animation Data Repository}

As the data resource, the animation digital assets are multimodal that involves audio, video, 2D image/textual, 3D models, motion files, scene files etc., and they vary a lot. Targeting different topics and depending on the specific application domains, the contents of the digital data repositories are also different. From a semantic perspective, the repository could be abstracted and analysed with several sublayers. Taking 3D contents for example, they are interpreted with four layers in this paper: Geometry, Structure, Appearance and Logic, as illustrated in Fig. 5 (a). Fig. 5 (b) presents an example of animation content, which is a digital character of the Chinese traditional shadow puppetry. The shape can be considered has a structure composed by different parts of shadow puppet presenting geometric information. Added with the appearance information, such as colour, pattern or accessories, a complex character is created carrying rich culture contents. As a higher level of expressiveness, it represents a brave warrior in the play of "The Romance of Three Kingdoms" from the aspect of the logic layer.

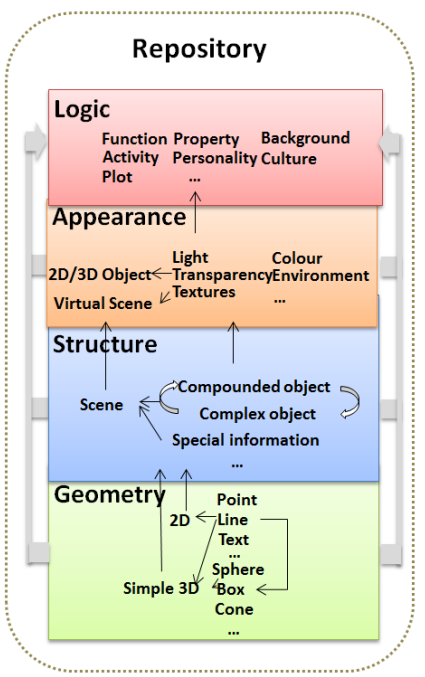

(a) 


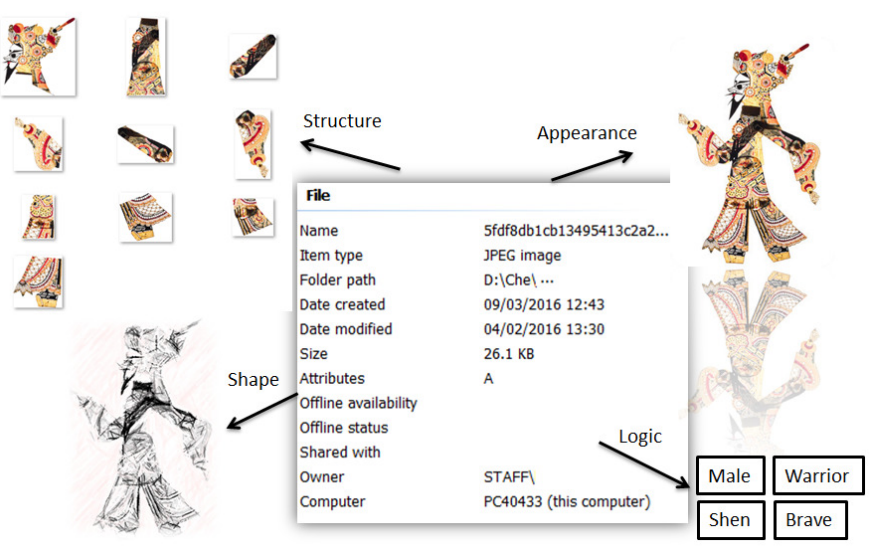

(b)

Fig. 5. Animation Data Repository component

The conceptual framework can systemise and standardise the procedure of interactive game/animation synthesis and promote system integration in a semantic representation. The two components are abstract concepts of interactive animation generation. Hence, the framework enables conceptual design for various interactive animation applications.

\section{USAGE EXAMPLE: HAND-GESTURE-BASED INTERACTIVE SHADOW PLAY ANIMATION}

Our framework sets a semantic foundation to formally model the interactive animation production from the abstract high level. This section focuses on investigating the cases in which we apply this semantic framework to guide the implementation of the domain specified ontologies. As a practical example, our method is presented in the context of a 2D Chinese traditional shadow puppetry performance scenario, in which players manipulate virtual characters to produce animation with hand gestures utilising depth motion sensing device - the Leap Motion controller.

\section{A. Ontology design}

Using the Web Ontology Language (OWL [39]) together with the ontology editor and knowledge framework-Protégé [40], the proposed semantic framework has been implemented as two domain specified ontologies: Hand-Gesture-Based Interaction Ontology (HGBIO) mapped to the HCI component, and Digital Chinese Shadow Puppetry Assets Ontology (DCSPAO) mapped to the Animation Data Repository component. We take advantage of the OWL ontology structure in order to incorporate SWRL (Semantic Web Rule Language) [41] rules, which can be visualised using SPARQL (a recursive acronym for SPARQL Protocol and RDF Query Language) [42] queries for knowledge access.

\section{1) Hand Gesture-Based Interaction Ontology (HGBIO)}

Traditional shadow puppet is made of several flat parts and linked by joints. In a shadow show, puppet is controlled by the puppeteer's hand with sticks attached to the puppet.

In our method, we adopt the traditional puppet design mode and each digital puppet consists of ten parts linked by nine joints. Digital puppet's motion is controlled by the player's hand movement and gesture. During playing, the player's hand position is mapped to the position of puppet in virtual environment through motion sensor after translating. The animation is generated through a hand-gesture-based interaction. The detail of the motion control is described in Sections B "Implementation".

The diagram of a part of the HGBIO is shown in Fig. 6 (a). The ontology defined beneath the root of every OWL ontology "owl:Thing" has three subclasses: "Human", "Device" and "Method". Each subclass (ancestor) contains various subclasses (descendant) of their own. Each one of these descendants contains individuals for the assignment of characteristics. A number of object properties are designed to give the ontology fundamental relational functionalities. The set of data type properties are provided to link OWL individuals with typical data values. The structure of hand gesture ontology is organised in a hierarchy consisting of three levels. The lower level presents motion tracking data accessed from the Leap Motion sensor controller including information of frames, list of detected hands and list of pointables (such as fingers or finger-like tools). Each frame contains the measured positions and the following information of the object detected by the controller. The device provides model of hands with five fingers of bone structures and each finger is made of four bone joints. By using the positions and rotations information of each finger bone we could design specific interactions. At the middle level, by calculating hands movement speed, the angle of movement, and the orientation angle, we can define simple hand gestures and describe the hand movement. For example, "Leftward" represents "Fly/run to the left"," Rightward" represents "Fly/run to the right", "Upward" represents "Fly/jump up", "Downward" represents "Fly/crouch down", and "Stay still" represents "Hover in the air/stay unmoved" etc. At the top level, we can develop more complex hand gestures which are the combination of simple hand gestures. These compound gestures could meet the player's further needs by depicting sophisticated interactive semantic meaning. For example, "Hand open to close" represents "Grasp", "Hand close to open" represents "Drop", and "rapid multiple finger taps" represents "Swipe wings" etc.

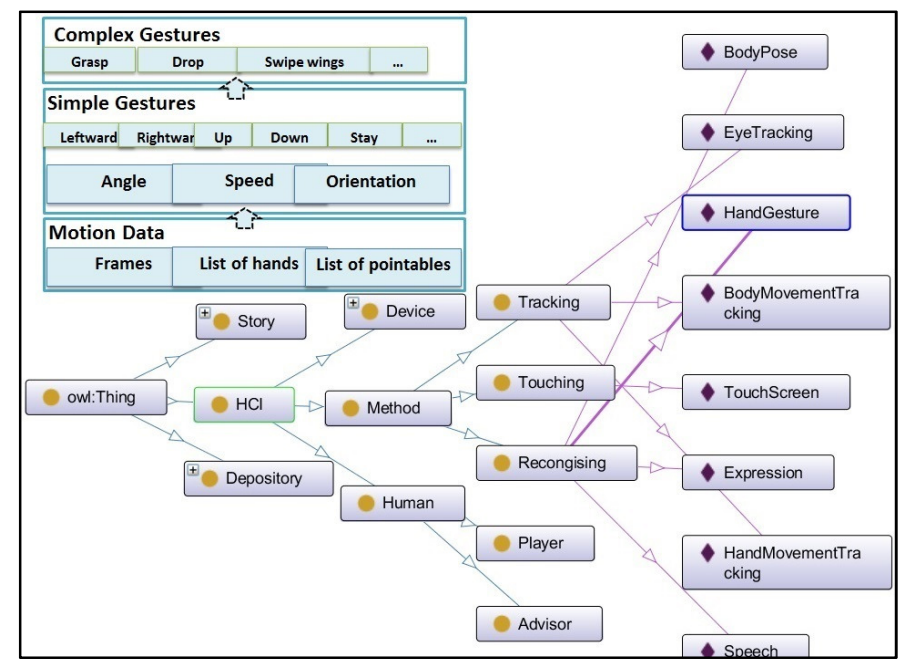

(a) 


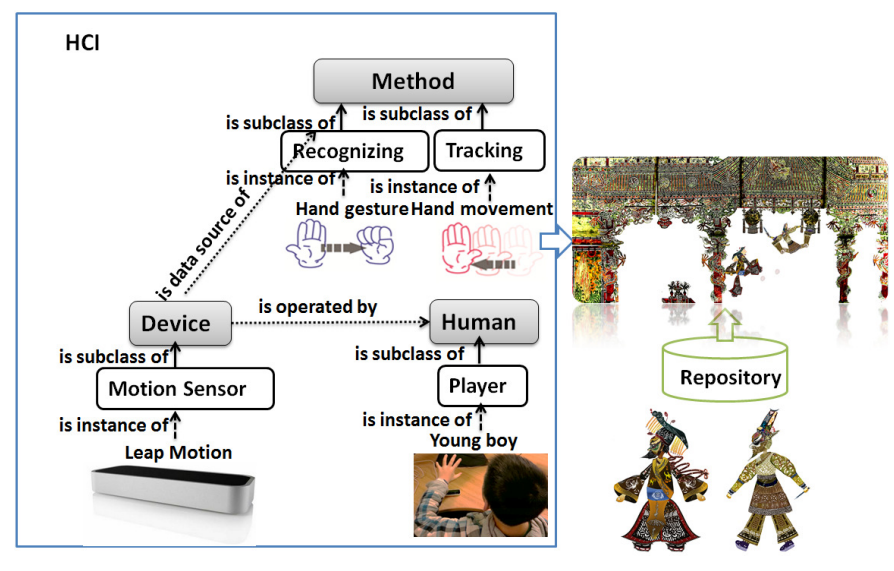

(b)

Fig. 6. Diagram of Hand-Gesture-Based Interaction Ontology

Fig. 6 (b) shows a usage example of the defined HGBIO. The visualization displays the relationships among the selected class and other classes in its neighbourhood.

"Player" is a subclass of "Human", which has an instance - a young boy who is using his hand gesture to control the movement of an avatar in the virtual environment through Leap Motion controller. Through the object property "is operated by", the Leap Motion controller is connected with player to deliver the semantic information: "Device is operated by play for interaction".

There are a range of interactive devices that enable user interactions using hand gestures, such as Leap Motion and Kinect etc. In this diagram, Leap Motion is an instance of class "Motion Sensor" which is a subclass of "Device". The essential attribute of Leap Motion is to provide data to the class "Method", which is described by the object property "is data source of" that links two classes.

The sensor data provided by Leap Motion contains a diversity of information about hands and fingers including id, direction, position, orientation, relative information and status etc. "Method" is the core of the system, which has two subclasses in our case: "Tracking" and "Recognizing". Hand Movement is an instance of "Tracking", which is responsible for processing the player's hand movement and mapping the relative hand position to the movement of the avatar in virtual world. Recognizing function is in charge of identifying the patterns of hand movement and gestures implicating the player's intention. Hand gesture is an instance of "Recognising", which provides a natural and intuitive gestural interaction manner. Once a recognisable hand gesture is detected, the recognition function will trigger a pre-recorded animation or event (e.g. avatar grips a pebble or flaps wings).

2) Digital Chinese Shadow Puppetry Assets Ontology (DCSPAO)

Traditional Chinese shadow play has distinctive folk style and artistic characteristics. As the implementation of the Domain Layer of the proposed semantic framework, a domain specified ontology is developed for traditional Chinese shadow puppetry, furthermore, a shadow puppetry animation data repository is also used as the digital assets for the animation generation.

The diagram of a part of the DCSPAO is shown in Fig.7 (a). Defined beneath the root of every OWL ontology "owl:Thing", DCSPAO has several subclasses including "Role", "Music", "Prop" and "Scene" etc. And each of these subclasses has the superclass at the same time, also its descendant. For example, the human characters in traditional Chinese shadow puppetry play fall into four major roles - Sheng (main male role), Dan (female role), Jing (painted-face and forceful male role), Chou (clown male role), which leads to four subclasses of the class "Role" correspondingly [43].

A set of object properties are added to create relationship among classes by linking relevant objects, such as "has Prop of", "has Scene of", "has Music of" etc. We also propose data properties which provide attribute descriptions for classes in the DCSPAO, including "name", "age", "rank" and "personality" etc.

As an example, an ontological description of the character "Guan Yu" is illustrated in Fig. 7 (b), who is one of the most famous general in ancient China. He is widely respected as a symbolic cultural icon of loyalty and righteousness in general worship. "Laosheng" (decent middle/old-aged man with beard) is a subclass of "Sheng" (main male role). As its instance, "Guan Yu" has a set of inherited properties including name, age, personality etc. As one of the most famous characters in shadow play, "Guan Yu" has unique personality and character symbolism, which is normally presented by particular props (e.g., his trademark weapon) and other dramatic elements. This specified relation between the class "Role" and the class "Prop" is determined by the object property which is defined in the domain ontology. For instance, through the object property "has Prop of", "Guan Yu" and his pole weapon named "Green Dragon Crescent Blade" (an instance of the subclass of "Weapon") are connected, which is the same as "Guan Yu" and his war horse named "Red Hare" (an instance of the subclass of "Animal" which belongs to other characters except human roles).

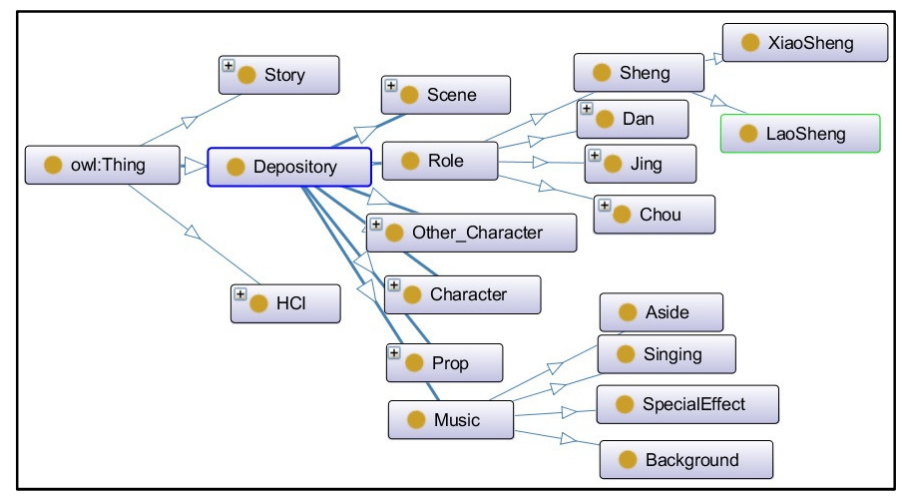

(a) 


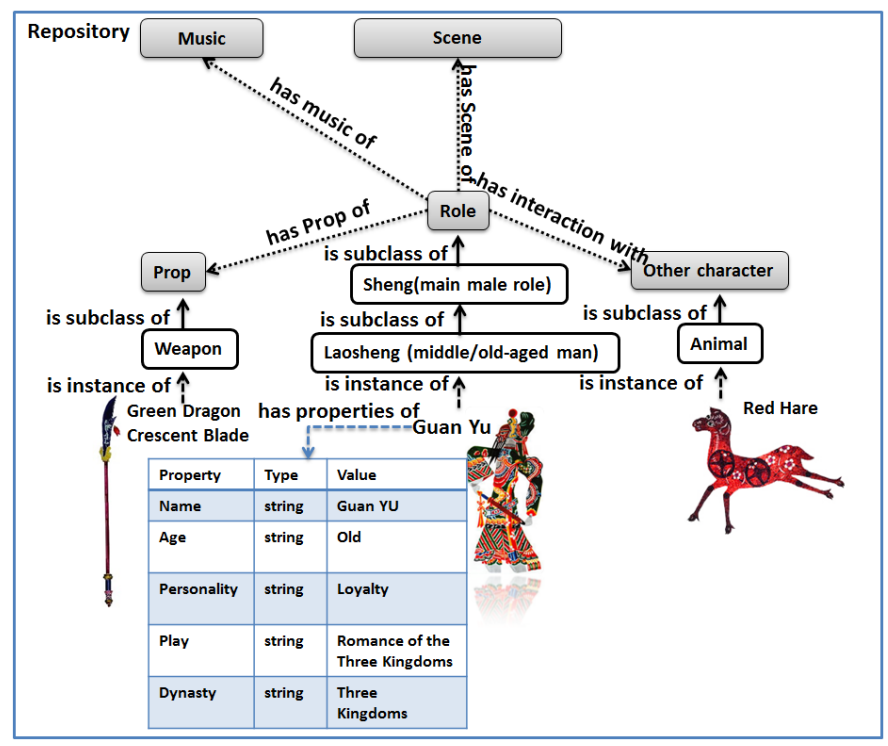

(b)

Fig. 7. Diagram of Digital Chinese Shadow Puppetry Assets Ontology

Since "Guan Yu" is a significant role in the classic shadow play "Romance of Three Kingdoms", object property "has Scene of" can assign a particular instance ("Military Pass") of the class "Scene" to him, which implicates the significant battlefield in the play. As a key role in shadow play, music creates particular atmosphere and shape characters. Object property "has Music of" can be used to reflect the bravery personality by connecting the unique melody "Splendour Allegro" with "Guan Yu".

\section{B. Implementation}

We discuss in the following how the proposed semantic Framework can be employed in order to inform the design of interactive animation. A practical example is presented in the context of a 2D Chinese traditional shadow puppetry performance, which enable players to interact with virtual shadow puppetry using hand gestural control.

As the ontological implementation of HCI component in the semantic framework, HGBIO has been used as a guidance to develop a novel hand-gesture-based interaction method. By utilizing depth motion sensing technology, the method tracks and recognises user's hand and finger motions as input, which enables the users to use hand gestures to control the movement and manipulate animation of the digital shadow puppetry with natural interaction/control and immersive experience. DCSPAO has been used to implement the Animation Data Repository component of the semantic framework. Using domain-specific information extraction, inference and rules to exploit the semantic metadata, the repository supports ontology-based retrieval, which improves searching performance by recognising the animator's intent and contextual meaning of the digital assets in the context of Chinese traditional shadow play. More relevant results can be generated to meet the user's needs and also provide the capability of data reuse. Fig. 8 gives an overview of the prototype architecture which has the following components:

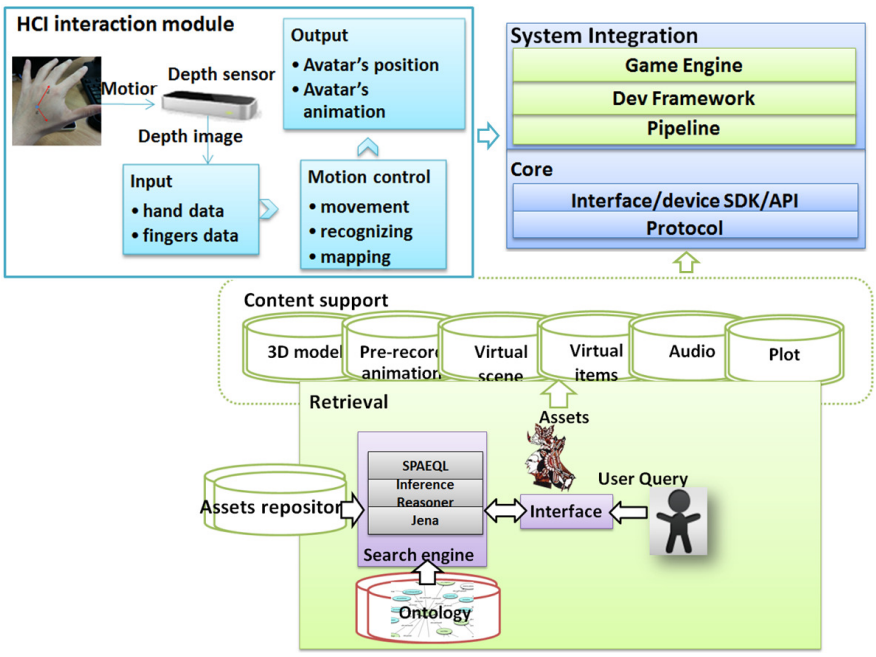

Fig. 8. Prototype architecture

HCI interaction module: We utilised a Leap Motion controller as the HCI sensor device to track hand gestures, which can provide a high fidelity finger tracking through an infrared depth sensor. We utilised the Leap Motion SDK API to access the motion data of hands and fingers from the device. Through movement control and recognition, the player's relative hand position and gestures are mapped to the movement of the digital shadow puppetry in the virtual environment.

HCI interaction module mainly includes three sub-modules: input data processing, movement control and output. For more details of hand movement control mechanism and gesture recognising, please refer [44-45].

Assets Repository: The repository is a mixed-type animation data database of digitalised traditional Chinese shadow puppets involving domain specified knowledge including classification of performers and roles, visual/aural performance elements and repertoire etc., which depicts model's high-level concept.

Retrieval system: assets retrieval includes a retrieval engine and a retrieval interface. Retrieval engine executes reasoning, inferring and querying to find assets which meet the players' needs. Retrieval interface as the visual front-end, receives artist's query and calls the engine for retrieval, and presents results and feedbacks with related information, e.g., the title and the abstract, plus information about the assets etc.

Through this ontology-based retrieval system which maps artist's intending on the data stored in the digital assets ontology, we can handle this domain related query and provide more reasonable and relevant feedback to meet the artist's needs and to fertilise data reuse.

Let's take the shadow puppet character "Jing Ke" as an instance to show all his relationship to other entities. "Jing Ke" is the hero of a famous Chinese traditional shadow play titled "The Emperor and the Assassin". A brave fighter named "Jing Ke" attempted to assassinate the king of Kingdom Qin - "Qin Shi Huang" to avert the imminent conquest of his home country by Kingdom Qin. After intense fighting in the palace, 
however, the assassination attempt failed, and "Jing Ke" was killed on the spot.

For the character designer of the play, there is plenty of animation data existing to be referred to and reused as digital assets in the shadow puppet repository. If he wants to review all the subjects and objects that related to the character "Jing Ke", through the object properties which are used to link to classes or individuals of another, such as "has Prop of", "has Scene of" and "has Music of", related entities (e.g., his weapon and the scene of the play etc. ) are retrieved.

The following SPARQL Query Language is used as inputs: "SELECT ?property ?object WHERE \{ base: Jing_Ke? property ?object\}".

The query will return a result that contains the set of all properties and objects that is related to the character "Jing Ke".

Pseudocode of using Jena API (a Java framework for building Semantic Web) [46] for querying from database is described as below:

// create a connection to database

IDBConnection conn=new DBConnection(DB_ URL , DB_USER , DB_PASSWORD);

//use connection to construct model (MODEL_NAME) in the database: ModelRDB $\mathrm{m}=$ ModelRDB.open(conn, MODEL_NAME);

//create shadow puppet assets ontology model spOntology

OntModel spOntology = ModelFactory. createOntologyModel();

//create OWLReasoner, and bind with spOntology

Reasoner owlReasoner=ReasonerRegistry.getOWLReasoner();

Reasoner spReasoner=owlReasoner.bindSchema(spOntology);

//create inferring model

InfModel infModel=ModelFactory. createlnfModel(spReasoner, model);

//Once a Query is prepared; it will be passed to QueryEngine to execute querying

String queryString=“...”;

Query query=new Query(queryString);

query.setSource(infModel);

QueryExecution qexec=new QueryEngine(query);

QueryResults results $=$ qexec $\operatorname{exec}()$;

The retrieval system is shown in Fig. 9. For instance, the artist inputs a key word "Jing Ke". Not only the digital character "Jing Ke" itself is retrieved, other useful assets related to the given object instance are also found from the knowledge base, which may satisfy the artist's requirements, such as his target "Qin Shi Huang", the scene affiliated to the play, and his weapon used in the story etc.

\section{Result}

Fig. 10 illustrates the digital shadow puppetry play "The Emperor and the Assassin" performed by a player. The heroes of the play "Jing Ke" and "Qin Shi Huang" are animated to fight with each other on the virtual stage. Their motion is generated by the player's hand gestures and movement through Leap Motion controller. The novel interaction provides player a more immersive and interactive experience and enable him perform the story in an intuitive and natural way. Screen shots of generated digital shadow play - "The Emperor and the Assassin" are shown in Fig. 11.

We have observed that it is relatively easy for the player to use one hand to control one character. But manipulating two puppets simultaneously, each by one hand, are rather challenging. This kind of operation demands bimanual coordination skills.

We hope this appealing interactive animation generation method may help people become intimate with their cultural heritage and promote traditional arts and culture among the young generation.

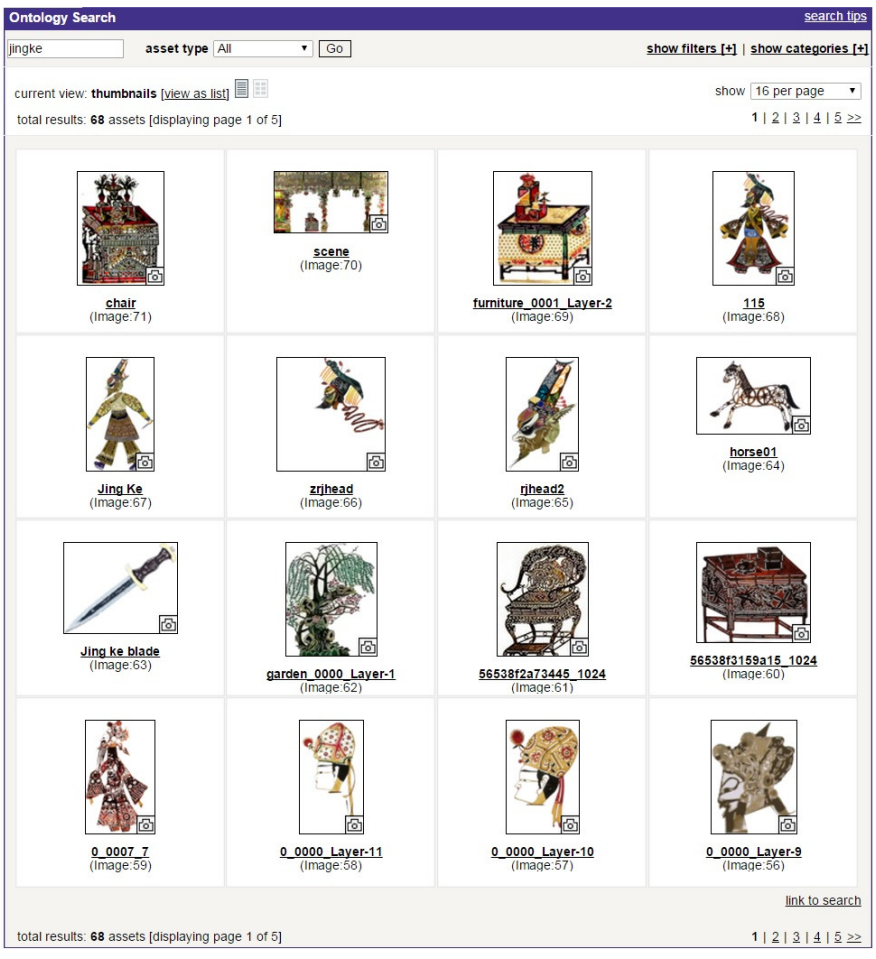

Fig. 9. Retrieval system

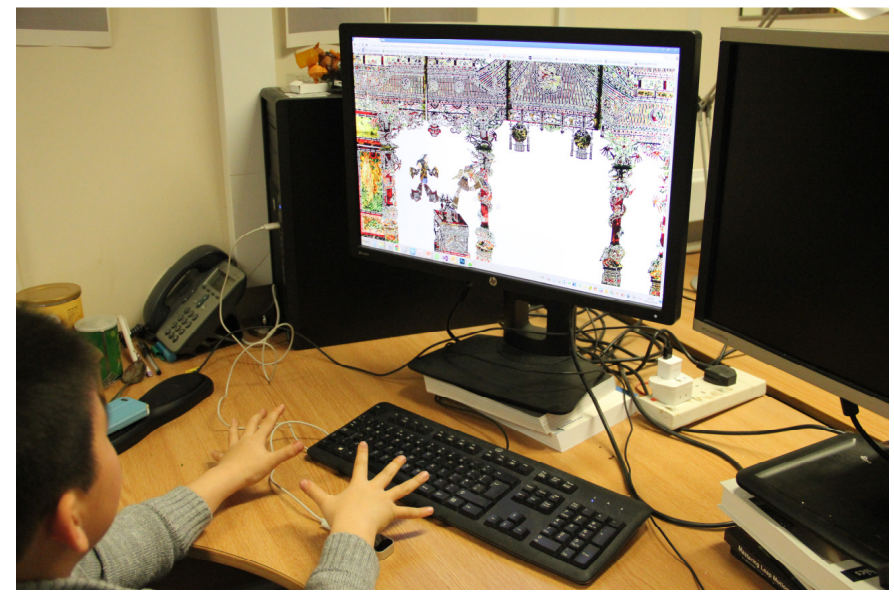

Fig. 10. Scenarioes of digital shadow play performed by a player 

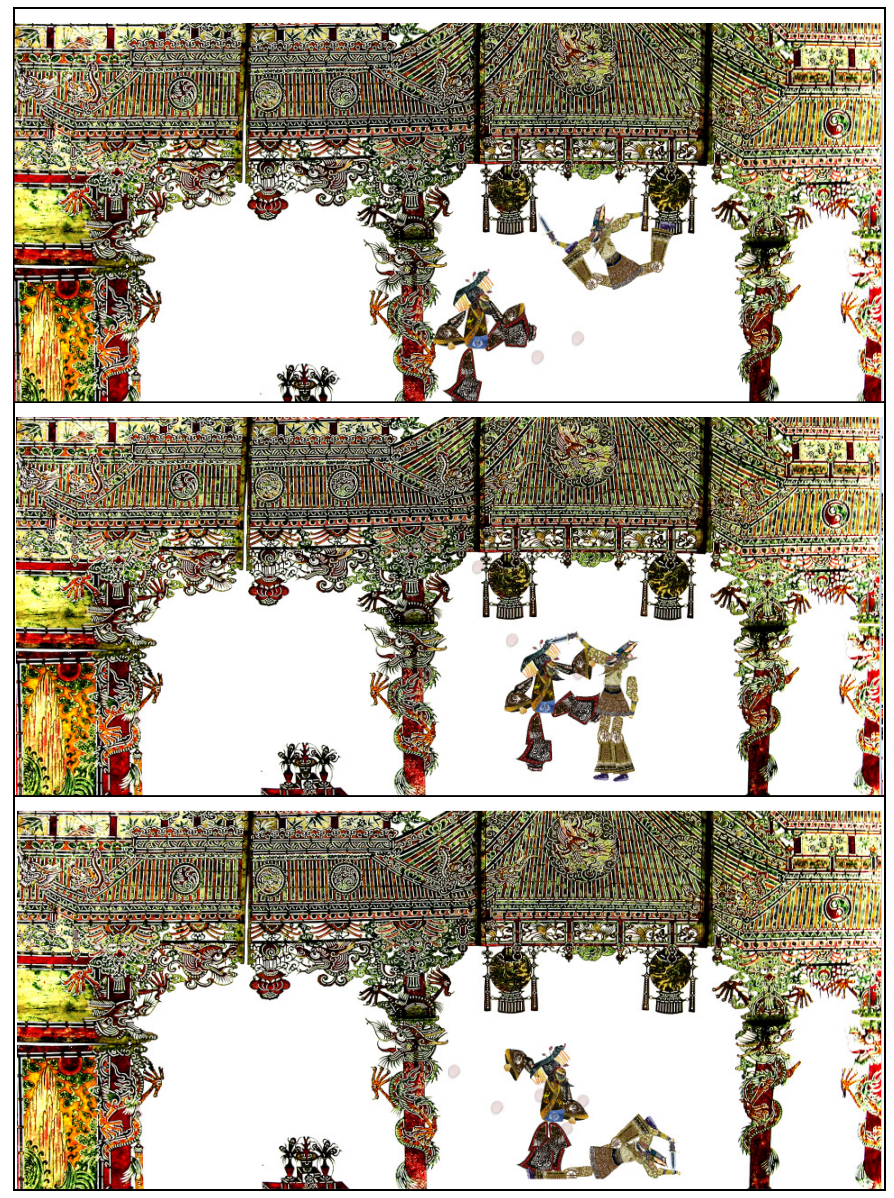

Fig. 11. Screen shots of digital shadow play "The Emperor and the Assassin"

\section{DISCUSSION AND CONCLUSION}

The construction process of interactive animation is complex because there are too many low-level details involved, for instance, multimedia animation data management and data reuse, user-friendly interaction and integration etc. To provide a systematic and standardised semantic description, a semantic framework is constructed at an abstract high-level in this paper. The ontological implementation based on the framework defined two domain specified ontologies (Hand-Gesture-Based Interaction Ontology and Digital Chinese Shadow Puppetry Assets Ontology) to formalise the multimodal interaction method and the construction of the animation data assets repository, which finally leads to an interactive puppetry animation as a usage example.

Ontology is the core element of our research, by using which we provided a concept depiction for the complex animation production process, derived a semantic representation providing a more profound understanding of HCI, and presented the specific domain knowledge of traditional Chinese shadow play art. Ontology is a high-level conceptual representation of the components in the animation production. The defined class hierarchy, object properties and data properties revealed how the low-level details involved in animation production are related to each other and how they collaborated and interacted with each other as well as the players and devices.
Our main goal is to utilise semantic/ontology concept to improve the reusability, extensibility and modularity of the interactive animation production and facilitate the development process. The proposed framework is flexible and extendable for many applications. In the usage example, we mainly worked with hand-gesture-based interactive digital shadow play, which provides a novel method to generate stylised traditional shadow play animation. This will contribute to the preservation of this cultural treasure of art. Based on the semantic framework, researcher can define various domain specified ontologies and construct animation asset repositories depending on the context and application.

\section{ACKNOWLEDGMENT}

The research leading to these results has received funding from the People Programme (Marie Curie Actions) of the European Union's Seventh Framework Programme FP7/20072013/ under REA grant agreement $n^{\circ}$ [623883] - "AniM". The authors acknowledge partial support from project Dr Inventor (FP7-ICT-611383) and AniNex (FP7-IRSES-612627).

\section{REFERENCES}

[1] F. Chen, "Visions for the masses: Chinese shadow plays from shaanxi and shanxi," Pacific Science 201, 2015.

[2] A. Ramaprasad, and S.S. Papagari, "Ontological design,” In Proceedings of the 4th International Conference on Design Science Research in Information Systems and Technology, p. 5. ACM, 2009.

[3] J. Floty'nski, and K.Walczak, "Conceptual semantic representation of 3d content," In Business Information Systems Workshops, pp. 244-257. Springer Berlin Heidelberg, 2013.

[4] J. Floty'nski, and K.Walczak, "Semantic modelling of interactive 3d content," In Proceedings of the 5th Joint Virtual Reality Conference, pp. 41-48. Eurographics Association, 2013.

[5] J. Floty'nski, and K.Walczak, "Semantic multi-layered design of interactive 3d presentations," In Computer Science and Information Systems (FedCSIS), 2013 Federated Conference on, pp. 541-548. IEEE, 2013.

[6] J. Floty'nski, and K.Walczak, "Multi-platform semantic representation of interactive $3 \mathrm{~d}$ content," In Technological Innovation for Collective Awareness Systems, pp. 63-72. Springer Berlin Heidelberg, 2014.

[7] Z. Li, and K. Karthik, "Ontology-based design information extraction and retrieval," AI EDAM: Artificial Intelligence for Engineering Design, Analysis, and Manufacturing, 21(2) , 2007, pp. 137-154.

[8] R. Ohbuchi, A. Yamamoto, and J. Kobayashi, "Learning semantic categories for 3D model retrieval," In Proceedings of the international workshop on Workshop on multimedia information retrieval, pp. 31-40. ACM, 2007.

[9] T.R. Gruber, "A translation approach to portable ontology specifications," Knowledge acquisition, 5(2), 1993, pp.199-220.

[10] M. Grüninger, and M.S. Fox, Methodology for the Design and Evaluation of Ontologies, 1995.

[11] W. N. Borst, Construction of engineering ontologies for knowledge sharing and reuse, Universiteit Twente, 1997.

[12] R. Studer, V. R. Benjamins, and D. Fensel, "Knowledge engineering: principles and methods," Data \& knowledge engineering, 25(1), 1998, pp.161-197.

[13] J.W. Tangelder and R.C. Veltkamp, "A Survey of Con-tent Based 3D Shape Retrieval Methods," Multimedia tools and applications, 39(3), 2008, pp.441-471.

[14] M. Kazhdan, T. Funkhouser, and S. Rusinkiewicz, "Rotation Invariant Spherical Harmonics Representation of 3D Shape Descriptors," In Symposium on geometry processing, vol. 6, pp. 156-164. 2003.

[15] B. Falcidieno, “Aim@ shape project presentation”, In Shape Modeling Applications, Proceedings, p. 329, IEEE, 2004. 
[16] B. Falcidieno, M. Spagnuolo, P. Alliez, E. Quak, M. Vavalis, and C. Houstis, "Towards the Semantics of Digital Shapes: The AIM@SHAPE Approach," In EWIMT, 2004.

[17] T. Funkhouser, P. Min, M. Kazhdan, J Chen, A. Halderman, and D. Dobkin et al., "A search engine for 3D models," ACM Transactions on Graphics (TOG) 22(1), 2003, pp. 83-105.

[18] Google 3D Warehouse, Available at: https://3dwarehouse.sketchup.com/ . Retrieved 21, Mar, 2016.

[19] Ontology for Media Resources 1.0. Available at: http://www.w3.org/TR/mediaont-10/. Retrieved 18, Mar, 2016.

[20] R. Arndt, R. Troncy, S. Staab, and L. Hardman, "Comm: A core ontology for multimediaannotation," In Handbook on Ontologies, pp. 403-421. Springer Berlin Heidelberg, 2009.

[21] M. Gutiérrez A., A. García-Rojas, D. Thalmann, F. Vexo, L. Moccozet, N. Magnenat-Thalmann, M. Mortara, and M. Spagnuolo, "An ontology of virtual humans: Incorporating semantics into human shapes," Vis. Comput., 23(3), pp. 207-218, 2007.

[22] K.A. Otto, "The semantics of multi-user virtual environments," In Proc. of the Workshop towards Semantic Virtual Environments, 2005.

[23] M. Gutierrez, D. Thalmann, and F. Vexo, "Semantic virtual environments with adaptive multimodal interfaces," In Multimedia Modelling Conference, 2005. MMM 2005. Proceedings of the 11th International, pp. 277-283, IEEE, 2005.

[24] D. Thalmann, N. Farenc, and R. Boulic, "Virtual human life simulation and database: why and how", In Database Applications in NonTraditional Environments, 1999.(DANTE'99) Proceedings. 1999 International Symposium on, pp. 471-479, IEEE, 1999.

[25] P.H.M. Chang, Y.H. Chien, E.C.C. Kao, and V.W. Soo, "A knowledge based scenario framework to support intelligent planning characters", In Intelligent Virtual Agents, pp. 134-145. Springer Berlin Heidelberg, 2005.

[26] I.M. Bilasco, J. Gensel, M. Villanova-Oliver, and H. Martin, "On indexing of 3D scenes using MPEG-7," In Proceedings of the 13th annual ACM international conference on Multimedia, pp. 471-474. ACM, 2005.

[27] H. Mansouri, "Using semantic descriptions for building and querying virtual environments," Licentiate's thesis, Vrije Universiteit Brussel, 2005.

[28] J. De Boeck, C. Raymaekers, and K. Coninx, "Comparing NiMMiT and data-driven notations for describing multimodal interaction," In Task Models and Diagrams for Users Interface Design, pp. 217-229. Springer Berlin Heidelberg, 2006.

[29] D. Ruminski, and K. Walczak, "Semantic contextual augmented reality environments," In Mixed and Augmented Reality (ISMAR), 2014 IEEE International Symposium on, pp. 401-404. IEEE, 2014.

[30] N. Mirbakhsh, A. Didandeh, and M. Afsharchi, "Concept Learning Games: The Game of Query and Response," In Web Intelligence and Intelligent Agent Technology (WI-IAT), 2010 IEEE/WIC/ACM International Conference on, vol. 2, pp. 234-238. IEEE, 2010.
[31] O.T. Leino, "What Erotic Tetris Has to Teach Serious Games About Being Serious? Design implications of an experiential ontology of game content," Meaningful Play 2010 Conference Proceedings, USA.

[32] J.S.F. Teixeira, E.D.J.V. Sá, and C. T. Fernandes, "A taxonomy of educational games compatible with the LOM-IEEE data model," Proceedings of Interdisciplinary Studies in Computer Science SCIENTIA, 2008, pp. 44-59.

[33] D. Dubin, and J. Jett, “An Ontological Framework for Describing Games," In Proceedings of the 15th ACM/IEEE-CE on Joint Conference on Digital Libraries, pp. 165-168. ACM, 2015.

[34] M. Anthony, T. Chandler, E. Heiser, "A Framework for Promoting High Cognitive Performance in Instructional Games," Interservice/Industry Training Simulation and Education Conference (I/ITSEC), Orlando, FL, 2009.

[35] A. BinSubaih, S. Maddock, and D. Romano, "Game logic portability," In Proceedings of the 2005 ACM SIGCHI International Conference on Advances in computer entertainment technology, pp. 458-461. ACM, 2005.

[36] J. Parkkila, T. Hynninen, J. Ikonen, J. Porras, and F. Radulovic, "Towards Interoperability in Video Games," In Proceedings of the 11th Biannual Conference on Italian SIGCHI Chapter, pp. 26-29. ACM, 2015.

[37] A. Ghannem, "Characterization of serious games guided by the educational objectives," In Proceedings of the Second International Conference on Technological Ecosystems for Enhancing Multiculturality, pp. 227-233. ACM, 2014.

[38] T. Tutenel, R.M. Smelik, R. Bidarra, and K.J. de Kraker, "Using Semantics to Improve the Design of Game Worlds," In AIIDE. 2009.

[39] World Wide Web Consortium, "OWL 2 web ontology language document overview," 2012.

[40] A free, open-source ontology editor and framework for building intelligent systems, Available at: http://protege.stanford.edu/ . Retrieved 21, Mar, 2016.

[41] SWRL: A Semantic Web Rule Language, Available at: https://www.w3.org/Submission/SWRL/. Retrieved 21, Mar, 2016.

[42] SPARQL Query Language for RDF, Available at: https://www.w3.org/TR/rdf-sparql-query//. Retrieved 21, Mar, 2016.

[43] Shadow Play, Available at: http://www.chinatraveldesigner.com/travelguide/culture/chinese-opera/shadow-play.htm Retrieved 21, Mar, 2016.

[44] H. Liang, J. Chang, I.K. Kazmi, J.J. Zhang, and P. Jiao, "Puppet Narrator: utilizing motion sensing technology in storytelling for young children," In Games and Virtual Worlds for Serious Applications (VSGames), 2015 7th International Conference on , pp. 1-8, IEEE, 2015.

[45] H. Liang, J. Chang, S. Deng, C. Chen, R Tong, and J.J. Zhang, "Exploitation of novel multiplayer gesture-based interaction and virtual puppetry for digital storytelling to develop children's narrative skills," in Proceeding VRCAI '15 Proceedings of the 14th ACM SIGGRAPH International Conference on Virtual Reality Continuum and its Applications in Industry , pp. 63-72, ACM, 2015.

[46] Apache Jena, Available at: https://jena.apache.org/ . Retrieved 21, Mar, 2016. 